\title{
A new autosomal recessive syndrome of characteristic facies, joint contractures, skeletal abnormalities, and normal development: second report with further clinical delineation
}

\author{
Ajay Gupta, Christine M Hall, Yvonne F Ransley, Victoria A Murday
}

\author{
Department of \\ Medical Genetics, \\ St George's Hospital \\ Medical School, \\ Cranmer Terrace, \\ London SW17 ORE, \\ UK \\ A Gupta \\ V A Murday \\ Department of \\ Radiology, Great \\ Ormond Street \\ Hospital and the \\ Insitute of Child \\ Health, Great Ormond \\ Street, London WC1N \\ 3JH, UK \\ C M Hall \\ Department of \\ Paediatrics, Epsom \\ General Hospital, \\ Dorking Road, Epsom, \\ Surrey KT18 7EG, UK \\ Y F Ransley \\ Correspondence to: \\ Dr Murday. \\ Received 4 May 1995 \\ Accepted for publication \\ 1 June 1995
}

\begin{abstract}
We describe a girl of Pakistani origin, born to consanguineous parents, with a multiple congenital anomaly (MCA) syndrome. This is the second report confirming an apparently new autosomal recessive syndrome reported earlier by van den Ende et al in 1992. The hallmarks of this MCA syndrome include characteristic facies with blepharophimosis, narrow, beaked nose, hypoplastic maxilla with or without cleft palate and everted lower lip, arachnodactyly, self-limiting congenital joint contractures, peculiar skeletal abnormalities, and normal growth and development. Further clinical and radiological delineation of the syndrome in this report suggests that the term "Marden-Walkerlike syndrome without psychomotor retardation", used in the first report to describe this condition, does not accurately reflect its clinical picture. The overall prognosis in this syndrome seems good.
\end{abstract}

( $\mathcal{F}$ Med Genet 1995;32:809-812)

Van den Ende $e t$ al $^{1}$ reported a Brazilian girl, born to a consanguineous couple, with a multiple congenital anomaly (MCA) syndrome characterised by blepharophimosis, cleft palate,
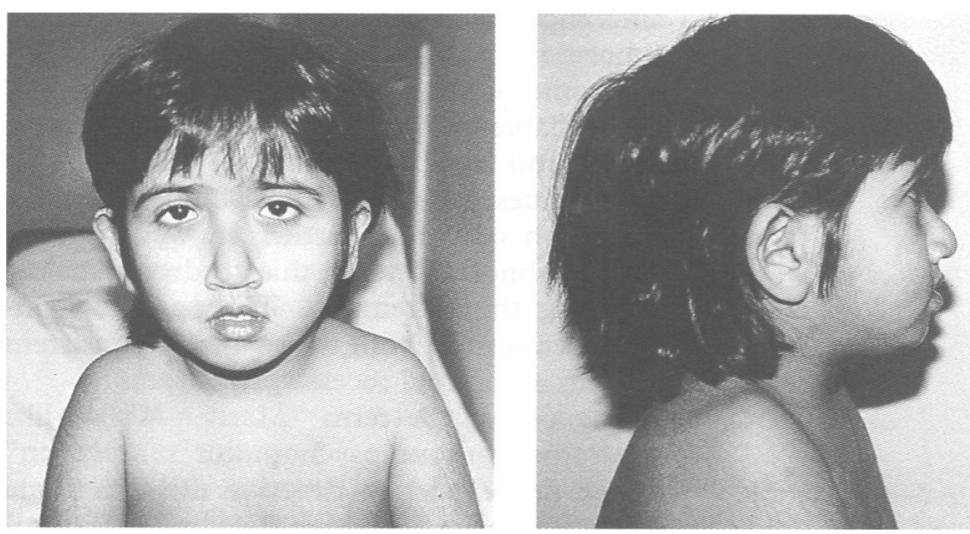

Figure 1 Front view and profile of the patient showing blepharophimosis, depressed nasal bridge, beaked nose, maxillary hypoplasia, and everted lower lip. and arachnodactyly. Her psychomotor development was normal. The authors described this entity as a "Marden-Walker-like syndrome without psychomotor retardation". We describe a girl of Pakistani origin, also born to consanguineous parents, with a similar MCA syndrome. However, further delineation of the clinical and radiological features indicate that, except for a few overlapping clinical signs, this apparently new autosomal recessive syndrome does not appear to be "like" the Marden-Walker syndrome (MWS).

\section{Case report}

The proband was born in October 1991 to healthy, consanguineous parents $(F=1 / 16)$ who were first cousins. She was the second of three daughters born to a 32 year old mother with no history of spontaneous miscarriages. The pregnancy was supervised and uneventful with no history of exposure to any known teratogen or illness. The baby was born at term by a normal vaginal delivery with a birth weight of $2950 \mathrm{~g}$. A single umbilical artery was noted at birth. The perinatal period was uncomplicated. Soon after birth, the baby was noted to have dysmorphic facies, long hands and feet, and symmetrical contractures mainly involving the distal joints of the extremities with marked stiffness and limitation of movements. Mild flexion contractures were also present at the knee and elbow joints bilaterally. There was no history of similar problems in her two sisters or in any of the close relatives. These flexion contractures gradually improved over a period of time without any intervention, and full range of movement is now possible at all the joints except the interphalangeal joints where mild contractures still persist without any significant functional impairment. There was some delay noted in her motor development during infancy, possibly because of the contractures, but thereafter her psychomotor development has been normal. She is described by her parents as a bright child who is more intelligent than her sisters. Her physical growth has been normal since birth with her weight, height, and 


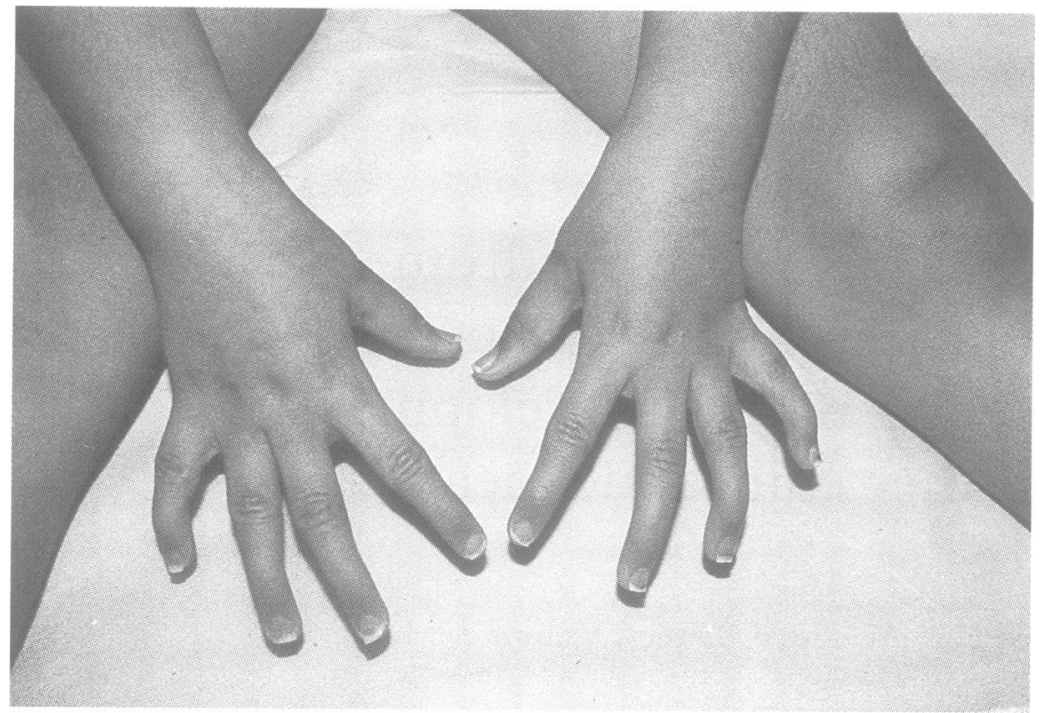

Figure 2 Hands of the patient with DIP camptodactyly of fingers 2-5, clinodactyly of finger 5 bilaterally, and hypoplastic nails.
The rest of her physical examination is within normal limits.

She has a normal karyotype $(46, \mathrm{XX})$. Fluorescent in situ hybridisation (FISH) study done to look for $22 \mathrm{q}$ deletion shows no apparent deletion in that region. Her urine amino acid chromatography and serum amino acid levels show a normal pattern. Results of her serum bone profile (calcium, phosphate, and alkaline phosphatase) are also normal. Owing to the presence of a single umbilical artery, an abdominal ultrasound was done at birth with normal results. After recovery from an episode of acute otitis media at the age of 2 years, audiometry showed normal hearing.

A skeletal survey done at the age of 2.5 years showed peculiar skeletal abnormalities (fig $4 \mathrm{~A}-$ G). In the skull, there is significant maxillary hypoplasia. The floor of the anterior cranial fossa slopes steeply and the anterior fossa is relatively small. Chest and shoulder joint $x$ rays show slender ribs, hooked lateral ends of the clavicles, absent glenoid fossae, and an unusual ossification centre of the coronoid process of the scapula bilaterally. In general, the long bones of the extremities are slender. In the upper limbs, the humeri are bowed laterally, the elbow joints show bilateral dislocation of the radial heads, the proximal ends of the radii and ulnae are deformed, and there is bowing and distal shortening of the ulnae. $X$ rays of her hands show normal bone age. The metacarpals and the proximal and middle rows of phalanges are long and slender, whereas the terminal phalanges are short and hypoplastic. The spine and pelvic bones are normal. In the lower limbs, the femora have long necks and are bowed laterally and the fibulae are thin and disproportionately long in relation to the tibiae. Her feet show abnormalities which are similar to the hands; in addition, there is an unusual triangular epiphysis of the terminal phalanx of the big toe giving rise to a valgus deformity bilaterally. feet and toes are also long with DIP camptodactyly of toes 2 to 5 , peculiar valgus deformities of the big toes at the terminal interphalangeal joints, an increased sandal gap, and hypoplastic nails (fig 3 ). There is restriction of movements at her elbow joints owing to bilateral dislocation of the radial heads (fig 4C).

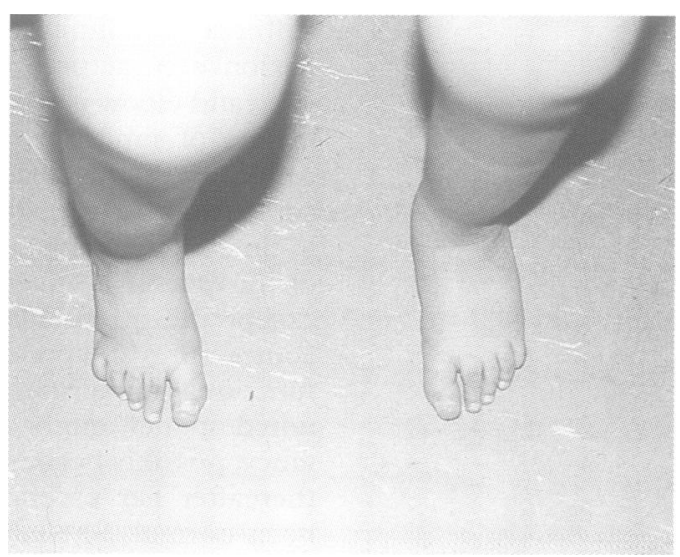

Figure 3 Feet of the patient showing DIP camptodactyly of toes 2-5, hypoplastic nails, and valgus deformity of the big toes at the terminal interphalangeal joints.

\section{Discussion}

The child described here bears a striking resemblance to the one reported earlier by van den Ende et al. ${ }^{1}$ Of the main clinical features described in the first report, only cleft palate is not present in our child, though she has a hypoplastic maxilla and a short palate. These differences may reflect the variability in the abnormal maxillary development present in this syndrome. Only $x$ rays of the hands and feet are available on the child described in the first report, and the abnormalities noted are similar to the ones present in this child. A skeletal survey on our child further showed peculiar skeletal abnormalities in this syndrome mainly involving the extremities. The pattern of inheritance seen in both the reports is consistent with an autosomal recessive syndrome.

The use of the term "Marden-Walker-like syndrome without psychomotor retardation" in the first report to describe this syndrome seems imprecise. ${ }^{1}$ Though the clinical features like blepharophimosis, arachnodactyly, and congenital joint contractures are present in both 


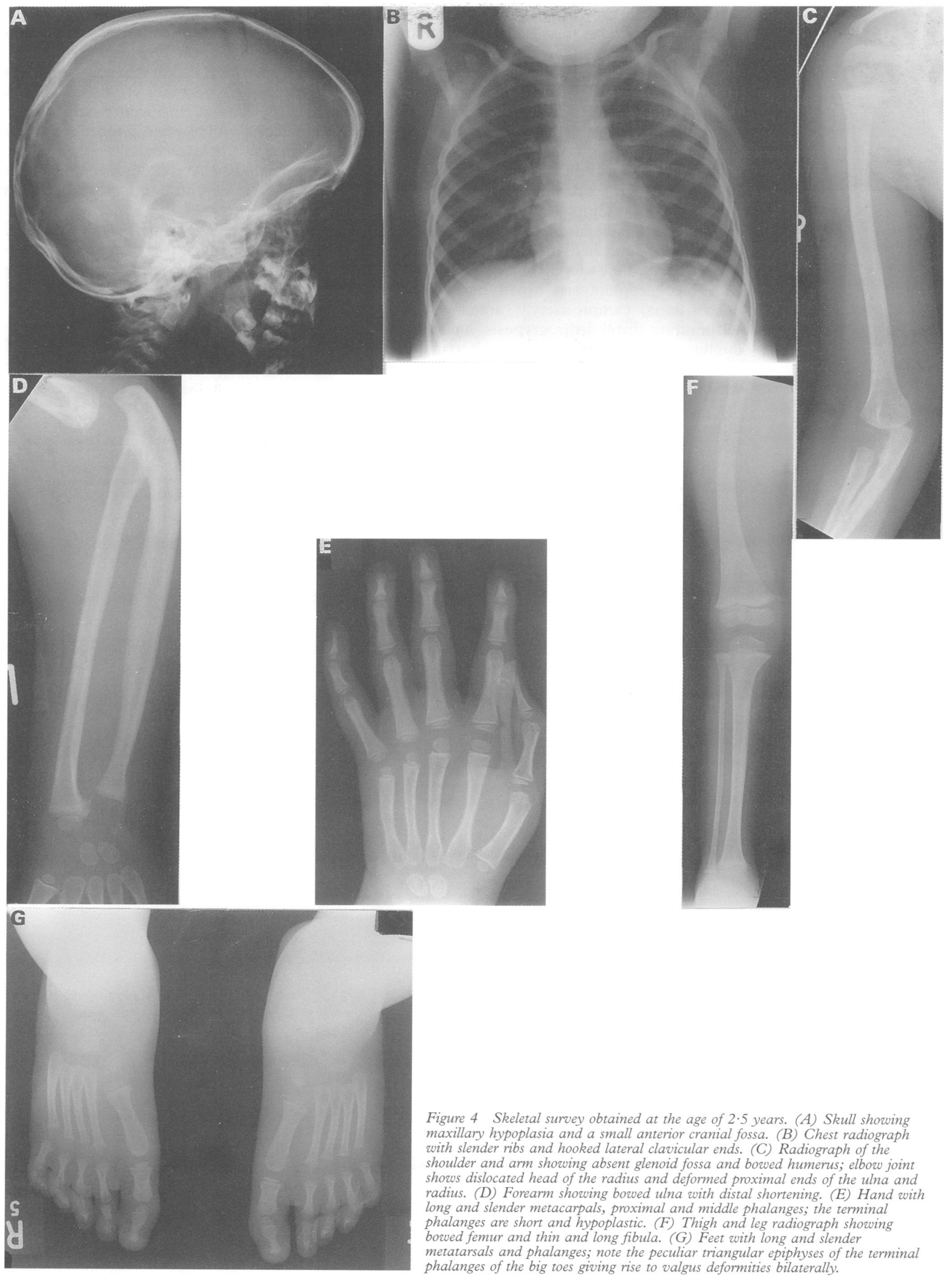

the MWS and this MCA syndrome, the two conditions have striking differences in their other clinical characteristics, radiological features, natural course, and prognosis. In the
MWS, all the cases published have severe psychomotor retardation, immobile, expressionless face, reduced muscle mass, and marked hypotonia; $95 \%$ of the cases also have 
severe postnatal growth retardation with failure to thrive, and more than $80 \%$ of them show low set/dysplastic ears and kyphoscoliosis. ${ }^{23} \mathrm{On}$ the other hand, the observations in this MCA syndrome show a characteristic dysmorphic facies, self-limiting distal joint contractures presenting with hypertonia in early infancy, a set of as yet unfamiliar skeletal abnormalities, and normal growth and development. The overall prognosis in this syndrome seems good.

Few other conditions can be considered in the differential diagnosis. There is a solitary case report published in the atlas of clinical syndromes, ${ }^{4}$ where a 9 year old girl was reported with blepharophimosis, broad nasal bridge, microstomia, camptodactyly, arachnodactyly, self-limiting distal arthrogryposis, and normal intelligence. However, she had short stature, webbed neck, and spinal column abnormalities which clearly differentiate her condition from the one reported here. In early infancy, conditions like Freeman-Sheldon and SchwartzJampel syndromes may mimic this entity owing to the lack of a full blown picture and the presence of distal joint contractures producing hypertonia, but other clinical features and the natural course in these two syndromes should be sufficiently characteristic to differentiate them from the condition reported here.

While confirming this apparently new autosomal recessive syndrome, we conclude that the main features of this syndrome are characteristic facies with blepharophimosis, narrow, beaked nose, hypoplastic maxilla with or without cleft palate and everted lower lip, arachnodactyly, congenital joint contractures, peculiar skeletal abnormalities, and normal growth and development. The clinical course observed in this child suggests gradual improvement in her congenital joint contractures with time.

1 van den Ende JJ, van Bever Y, Rodini ESO, Richieri-Costa A. Marden-Walker-like syndrome without psychomotor retardation: report of a Brazilian girl born to consanguineous parents. Am f Med Genet 1992;42:467-9.

2 Giacoia GP, Pineda R. Expanded spectrum of findings in Marden-Walker syndrome. Am $\mathcal{F}$ Med Genet 1990;36:4959.

3 Williams MS, Josephson KD, Wargowski DS. Marden-Walker syndrome: a case report and a critical review of the literature. Clin Dysmorphol 1993;2:211-9.

4 Weidemann HR, Grosse KR, Dibbern H. Das charakteristische Syndrom: Blickdiagnose von Syndromen. Ein Atlas fur Klink und Praxis. 3rd ed. Stuttgart: Schattauer Verlag, 1985: 382-3. 